\title{
Airborne Isocyanate Exposures in the Collision Repair Industry and a Comparison to Occupational Exposure Limits
}

\author{
Carolyn Reeb-Whitaker ${ }^{1}$, Stephen G. Whittaker ${ }^{1,2}$, Diana M. Ceballos ${ }^{3}$, Elisa C. Weiland ${ }^{4}$, \\ Sheila L. Flack ${ }^{5}$, Kenneth W. Fent ${ }^{5}$, Jennifer M. Thomasen ${ }^{5}$, Linda G. Trelles Gaines ${ }^{5}$, and \\ Leena A. Nylander-French ${ }^{5}$ \\ ${ }^{1}$ Safety \& Health Assessment \& Research for Prevention (SHARP) Program, Washington State \\ Department of Labor \& Industries, Tumwater, Washington \\ ${ }^{2}$ Local Hazardous Waste Management Program, Public Health—Seattle \& King County, Seattle, \\ Washington \\ ${ }^{3}$ University of Washington, Department of Environmental and Occupational Health Sciences, \\ Seattle, Washington \\ ${ }^{4}$ Division of Occupational Safety and Health Industrial Hygiene Laboratory, Washington State \\ Department of Labor \& Industries, Olympia, Washington \\ ${ }^{5}$ Department of Environmental Sciences and Engineering, University of North Carolina at Chapel \\ Hill, Chapel Hill, North Carolina
}

\begin{abstract}
Isocyanate exposure was evaluated in 33 spray painters from 25 Washington State autobody shops. Personal breathing zone samples $(n=228)$ were analyzed for isophorone diisocyanate (IPDI) monomer, 1,6-hexamethylene diisocyanate (HDI) monomer, IPDI polyisocyanate, and three polyisocyanate forms of HDI. The objective was to describe exposures to isocyanates while spray painting, compare them with short-term exposure limits (STELs), and describe the isocyanate composition in the samples. The composition of polyisocyanates (IPDI and HDI) in the samples varied greatly, with maximum amounts ranging from up to 58\% for HDI biuret to $96 \%$ for HDI isocyanurate. There was a significant inverse relationship between the percentage composition of HDI isocyanurate to IPDI and to HDI uretdione. Two 15-min STELs were compared: (1) Oregon's Occupational Safety and Health Administration (OR-OSHA) STEL of $1000 \mu \mathrm{g} / \mathrm{m}^{3}$ for HDI polyisocyanate, and (2) the United Kingdom's Health and Safety Executive (UK-HSE) STEL of $70 \mu \mathrm{g} \mathrm{NCO} / \mathrm{m}^{3}$ for all isocyanates. Eighty percent of samples containing HDI polyisocyanate exceeded the OR-OSHA STEL while $98 \%$ of samples exceeded the UKHSE STEL. The majority of painters $(67 \%)$ wore half-face air-purifying respirators while spray painting. Using the OROSHA and the UK-HSE STELs as benchmarks, $21 \%$ and $67 \%$ of painters, respectively, had at least one exposure that exceeded the respirator's OSHA-assigned protection factor. A critical review of the STELs revealed the following limitations: (1) the OR-OSHA STEL
\end{abstract}


does not include all polyisocyanates, and (2) the UK-HSE STEL is derived from monomeric isocyanates, whereas the species present in typical spray coatings are polyisocyanates. In conclusion, the variable mixtures of isocyanates used by autobody painters suggest that an occupational exposure limit is required that includes all polyisocyanates. Despite the limitations of the STELs, we determined that a respirator with an assigned protection factor of 25 or greater is required to protect against isocyanate exposures during spray painting. Consequently, half-face air-purifying respirators, which are most commonly used and have an assigned protection factor of 10 , do not afford adequate respiratory protection.

\section{Keywords}

autobody; hexamethylene diisocyanate; occupational exposure limits; polyisocyanates; respiratory protection; STEL

\section{INTRODUCTION}

Painters and other production workers in the collision repair industry are potentially exposed to a variety of chemical hazards, including isocyanates. ${ }^{(1)}$ Isocyanates are a major component of automotive coatings. In polyurethane-based coating systems, isocyanates are present in the catalyst hardener component of the formulation. Generally, the final "clearcoat" is recognized as the principal concern, reflecting the relatively high concentrations of isocyanates in the hardener. The most common isocyanates used in clearcoats are 1,6-hexamethylene diisocyanate (HDI) monomer and its polyisocyanate derivatives (i.e., uretdione, biuret, and isocyanurate). Some formulations may also contain monomeric and polyisocyanate forms of isophorone diisocyanate (IPDI). Typically, the monomers represent less than $5 \%$ of the hardener formulation, while the remainder is composed of HDI polyisocyanates and, occasionally, IPDI polyisocyanates.

Spray application of automotive coatings creates an over-spray of unreacted and partially reacted isocyanates, which represent a significant inhalation hazard. ${ }^{(2)}$ Dermal exposure may also occur during handling of paint components, paint mixing, spray application, cleanup, and handling of materials contaminated with partially polymerized product. ${ }^{(3)}$ In this study, the term "isocyanates" refers collectively to monomers and polyisocyanates. Elsewhere in the literature, polyisocyanates may be referred to as "oligomers."

Isocyanates are strong irritants and sensitizers ${ }^{(4)}$ and both the monomeric diisocyanate forms and polyisocyanates have been implicated in human respiratory sensitization. ${ }^{(5-9)}$ The epidemiologic data describing the burden of isocyanate-induced work-related asthma have been described. ${ }^{(10,11)}$ The American Thoracic Society estimated that $15 \%$ of adult asthma cases may be attributable to occupational exposure ${ }^{(12)}$ and isocyanates have been implicated as one of the most common causative agents of work-related asthma. ${ }^{(13-15)}$ Skin contact with isocyanates has been observed to induce systemic respiratory sensitization in animals. ${ }^{(16-19)}$

Two approaches are currently used to express occupational exposure limits (OELs) for isocyanates. The most common approach is to describe the OEL for individual isocyanates 
as either parts per million (ppm) or micrograms per cubic meter of air $\left(\mu \mathrm{g} / \mathrm{m}^{3}\right)$. The OELs for the monomeric forms of 2,4- or 2,6- toluene diisocyanate (TDI), 4, 4'-diphenylmethane diisocyanate (MDI), HDI, and IPDI are expressed in this manner by OSHA, the National Institute for Occupational Safety and Health (NIOSH), and ACGIH®. OELs have not been established for individual polyisocyanates, even though the coatings used in collision repair are primarily composed of polyisocyanates, such as HDI biuret, HDI uretdione, HDI isocyanurate, and IPDI polyisocyanate.

The most applicable OEL for the polyisocyanate mixtures used in collision repair is the Oregon OSHA (OR-OSHA) administration's short-term exposure level (STEL15min) of $1000 \mu \mathrm{g} / \mathrm{m}^{3}$ for HDI polyisocyanates. The OR-OSHA STEL $15 \mathrm{~min}$, promulgated in 1986 , is derived from animal toxicity studies with exposure to HDI isocyanurate and HDI biuret. ${ }^{(20,21)}$ The toxicity of HDI uretdione and IPDI polyisocyanate, which are common constituents in automotive coatings, were not part of the toxicity studies at the time the OROSHA STEL $_{15 \min }$ was established. ${ }^{(21)}$

The second approach to express OELs enumerates the total number of nitrogen-carbonoxygen (NCO) groups within an isocyanate mixture, rather than quantifying each individual species. This OEL is expressed as the mass of total $\mathrm{NCO}$ groups or as " $\mu \mathrm{g} \mathrm{NCO} / \mathrm{m}^{3}$." United Kingdom Health and Safety Executive's (UK-HSE) STEL 15 min standard for total NCO is 70 $\mu \mathrm{g} \mathrm{NCO} / \mathrm{m}^{3}$ for all isocyanates. ${ }^{(22)}$ The UK-HSE STEL 15 min was established in 1983 and includes monomers and polyisocyanates of all isocyanates, regardless of their individual identities or toxicities. ${ }^{(23)}$ The UK-HSE STEL 15 min is derived from the ACGIH's threshold limit value (TLV®, STEL ${ }_{15 \mathrm{~min}}$ ) for monomeric TDI, which is $0.02 \mathrm{ppm}$ (equivalent to 142 $\mu \mathrm{g} / \mathrm{m}^{3}$ with $48 \%$ of molecular weight as NCO). ${ }^{(23)}$ Because the UK-HSE STEL 15 min is based on the toxicological characteristics of TDI monomer, it may not be directly applicable to exposures occurring during automotive spray applications, where the predominant isocyanate species in the paint products are polyisocyanates of HDI and IPDI.

The goals of this study were to (1) measure the breathing zone concentrations of isocyanates during automotive spray painting; (2) compare measured isocyanate concentrations to the OR-OSHA STEL $15 \mathrm{~min}$ for HDI polyisocyanate and the UK-HSE STEL ${ }_{15 \mathrm{~min}}$ for all isocyanates; (3) evaluate the adequacy of protection afforded by painters' respirators; (4) describe the variability of isocyanate compositions in automotive coatings; and (5) describe the performance and variation of paint booths used in spray applications.

\section{METHODS}

\section{Painter Recruitment and Shop Characterization}

Automotive spray painters in the Puget Sound region of Washington State were recruited to participate in an exposure assessment study. Painters were recruited as described previously. ${ }^{(24)}$ Recruitment, sampling, and data handling procedures were approved by the Washington State Institutional Review Board (WSIRB) at the Washington State Department of Social and Health Services, and by the Institutional Review Board in the Office of Human Research Ethics at the University of North Carolina at Chapel Hill. The exposure assessments were conducted between August 2006 and February 2007. A total of 33 painters 
were recruited from 25 autobody shops. One large shop had three painters, six shops had two painters each, and 18 shops had one painter each enrolled in the study. Although initially we aimed to sample all 33 painters on three separate occasions (i.e., a repeated measures study design), attrition was such that eight painters were monitored twice and one painter monitored only once. Painters were lost to the study due to shop closure, job change, or personal leave of absence. Information concerning the use of respiratory protection and other industrial hygiene data was recorded during each sampling visit. A summary of the dermal protection worn by painters in this study is reported by Ceballos et al. ${ }^{(25)}$

\section{Air Sampling}

Personal breathing-zone concentrations were measured whenever a painter applied an isocyanate-containing coating using a spray gun. To determine whether isocyanates were present in a product, the painter was consulted, container labels were reviewed, and material safety data sheets (MSDSs) were obtained prior to spray application. Isocyanate air sampling cassettes were made and analyzed by the Washington State Labor and Industries' Laboratory. The dual-stage closed-face cassettes made in-house were identical to commercially available ISO-CHEK samplers (SKC Omega Specialty Division, Houston, Texas). The first (aerosol) filter (37-mm, 5.0 um polytetrafluoroethylene; PTFE, SKC Inc., Eighty Four, Pa.) was derivatized in the field with 1-(2-methoxyphenyl) piper-azine (MOPIP, $1.0 \mathrm{mg} / \mathrm{mL}$; Sigma-Aldrich, St. Louis, Mo.) in reagent grade toluene and analyzed for polyisocyanates. The second (vapor) filter (37-mm glass fiber, SKC) was preimpregnated with 9- $\mathrm{N}$-methylaminomethyl anthracene (MAMA; Sigma-Aldrich) and diluted in the analytical laboratory with reagent grade methylene chloride before being analyzed for isocyanate monomers. Air sampling was conducted at $2 / \mathrm{L}$ min airflow and was initiated prior to the painter entering the spray booth and stopped when the painter exited the spray booth. Research staff recorded the actual time spent spraying (i.e., paint time $t$ ), which was used to calculate isocyanate concentration during the spray painting task.

The analytical methods used were capable of chromatographically resolving derivatives of HDI and IPDI monomers and polyisocyanates and are additionally described by Ceballos et al. ${ }^{(26-28)}$ High-pressure liquid chromatography (1100 HPLC series; Agilent Technologies, Santa Clara, Calif.), with diode array detection and fluorescence detection, was used. Both the monomer and polyisocyanate analyses used a reverse-phase column at room temperature (hypersil ODS $5 \mu \mathrm{m}, 200 \mathrm{~mm}, 2.1 \mathrm{~mm}$ I.D with guard column, Agilent). The chromatographic elution was different for the monomer and polyisocyanate analysis. The monomer derivatives were eluted with a flow of $0.3 \mathrm{~mL} / \mathrm{minute}(\mathrm{mL} / \mathrm{min})$ in two steps. In Step 1, 60\% acetonitrile with 40\% $215 \mathrm{mM}$ triethyl amine (TEAP, pH 3) was held for 24 min. In Step 2, this was then ramped over 1 min to $70 \%$ acetonitrile with $30 \%$ TEAP and held for $23 \mathrm{~min}$. The polyisocyanate derivatives were eluted in a gradient: initially $40 \%$ acetonitrile with 60\% TEAP held for $14 \mathrm{~min}$; then ramped slowly over 6 min to 50\% acetonitrile/50\% TEAP; then ramped over $8 \mathrm{~min}$ to $80 \%$ acetonitrile/20\% TEAP; and then held at $80 \%$ acetonitrile $/ 20 \%$ TEAP for 16 min. Finally, the program ended with a 2-min ramp to $40 \%$ acetonitrile with $60 \%$ TEAP. 
The calibration standards are summarized in Table I. HDI and IPDI monomer commercial standards, as well as diluted HDI and IPDI polyisocyanate bulk products from Bayer Material Science (Leverkusen, Germany), were used in calibration. The analysis parameters such as limit of detection (LOD), limit of quantitation (LOQ), recovery, and precision for the analytes are summarized in Table I. The LODs were based on a detectable standard or spiked media sample that did not meet the LOQ requirements. The LOQs were based on spiked media with an average acceptable recovery of $\geq 75 \%$. Overall recovery was expressed as an average of the percent recoveries established at five concentration levels having six replicates each. There was a bias for HDI uretdione recovery, which could result in overestimation of this species. While the source of the bias is unclear, it may be caused by the presence of impurities in the standards (bulk products) or by solvents used during sample preparation that affected the uretdione's response. The overall assay precision was calculated as 1.96 multiplied by the total coefficient of variation (CV).

Quality control was performed to ensure that the standard concentrations for HDI and IPDI monomer were within $\pm 15 \%$ of the theoretical value. Calibration checks (three to five concentrations) were run before, during, and after all sample analyses. The number of field and laboratory blanks run per sample set was at least $10 \%$ of the total number of samples for each type of blank.

\section{Data Management and Analysis}

The analytical laboratory provided results in units of mass $(\mu \mathrm{g})$ per filter for each isocyanate species. Following the procedures described by Hornung and Reed, ${ }^{(29)}$ an analyte mass below the LOD or the LOQ was assigned surrogate values by dividing the respective limits by $\sqrt{ } 2$. Because the analytical method identified each individual isocyanate species, it was possible to calculate the percentage fraction of each species measured within a given sample. The total HDI polyisocyanate air concentration (Table II), expressed as $\mu \mathrm{g} / \mathrm{m}^{3}$ timeweighted averaged (TWA) over $15 \mathrm{~min}$, was calculated using Eq. 1:

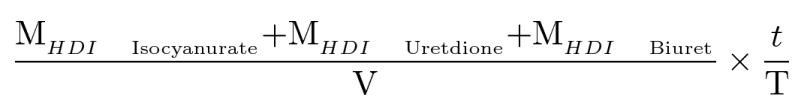

where $\mathrm{M}=$ mass, $\mathrm{V}=$ air-sample volume, $t$ paint time, and $\mathrm{T}=\min$.

The Total HDI Polyisocyanate NCO and Total All Isocyanates NCO expressed as $\mu \mathrm{g} / \mathrm{m}^{3}$ NCO STEL over 15 min (Table III) was calculated using Eq. 2:

$$
\frac{\sum_{i=1}^{5} \frac{\mathrm{M}_{i}}{\mathrm{~F}_{i N C O}}}{\mathrm{~V}} \times \frac{t}{\mathrm{~T}}
$$

where $\mathrm{M}=$ mass and $\mathrm{F}=\mathrm{NCO}$ correction factor as follows:

$$
\begin{aligned}
& \mathrm{M}_{1}\left(\mathrm{~F}_{1 \mathrm{NCO}}\right)=\text { HDI isocyanurate }(\mathrm{F}=4.64) \\
& \mathrm{M}_{2}\left(\mathrm{~F}_{2} \mathrm{NCO}\right)=\text { HDI uretdione }(\mathrm{F}=4.62) \\
& \mathrm{M}_{3}\left(\mathrm{~F}_{3} \mathrm{NCO}\right)=\text { HDI biuret }(\mathrm{F}=4.55) \\
& \mathrm{M}_{4}\left(\mathrm{~F}_{4} \mathrm{NCO}\right)=\text { IPDI polyisocyanate }(\mathrm{F}=5.79)
\end{aligned}
$$




$$
\mathrm{M}_{5}\left(\mathrm{~F}_{5} \mathrm{NCO}\right)=\text { HDI monomer }(\mathrm{F}=2.0)
$$

Samples for which the paint time was greater than 15 min were not time weighted. If $t>15$ $\mathrm{min}$, then the operation " $t / \mathrm{T}$ " was deleted from Equations 1 and 2 . The paint time was an average of $40 \pm 17$ percent (range 8 to 87 ) of the total pump run time and lasted an average of $8.1 \pm 6.3 \mathrm{~min}$ (range 1 to $53 \mathrm{~min}$ ). The maximum use concentrations (MUC, Table II, and Table III) for the respirator worn were calculated by multiplying the applicable STEL by the respirator's OSHA-assigned protection factor (APF). ${ }^{(30)}$

Repeated Measures Statistics—Variability statistics were calculated for HDI isocyanurate using the methods suggested by Rappaport et al. ${ }^{(31)}$ Variability and the Waldtype statistic were not calculated for the other analytes because their concentrations were imputed for $26 \%$ to $92 \%$ of the samples, depending on the analyte. The log-transformed HDI isocyanurate measurements fit a normal distribution ( $p>0.05$ ). To assess the fit of the one-way analysis of variance (ANOVA) with random effects model, a graphic p-p plot of the expected and observed cumulative distribution functions (CDFs) was generated with error bands of \pm 1 SD of the expected CDF. Review of the p-p plot revealed that the data were monomorphic and therefore acceptable for subsequent testing. The Wald-type statistic, as defined in Eq. 3 in Rappaport et al., was used to determine the probability that a randomly chosen worker's mean exposure $(\theta)$ is greater than the STEL. ${ }^{(31)}$ The hypothesis tested was $\mathrm{H}_{\mathrm{o}}: \theta \geq A$, where $A$, the maximum acceptable probability that a randomly chosen worker's mean exposure is greater than the STEL, was set at 0.10. Calculations were performed in Excel (Microsoft Corp., Redmond, Wash.) and in SPSS version 14.0.0 (SPSS Inc., Chicago, Ill.). Data were managed according to WSIRB requirements and kept confidential.

\section{Booth Ventilation}

Booth ventilation was evaluated at the exhaust grills using a rotating-vane anemometer (Model RVA501; ALNOR Instruments, Huntington Beach, Calif.) with an accuracy of \pm 20 linear feet per min (LFM, or six linear meters per min, LMM). The exhaust grills were located on the floor of downdraft booths and on the walls of semi-downdraft and cross-draft booths. A minimum of nine measurements were taken in a transect pattern across the grill area, with the anemometer held approximately one inch from the exhaust grills. The total cubic feet per minute (CFM or $\mathrm{Q}$ ) was calculated using the equation $\mathrm{Q}=\mathrm{VA}$ for standard exhaust banks, where $\mathrm{V}=$ velocity and $\mathrm{A}=$ area of exhaust bank. ${ }^{(33)}$ Two downdraft booths had slots in the floor with an aspect ratio (width/length) less than 0.2 . The equation $Q=2.6$ $\mathrm{LVX}$, where $\mathrm{L}=$ slot length, $\mathrm{V}=$ velocity, and $\mathrm{X}=$ distance between the anemometer and the exhaust slot was used to calculate $\mathrm{Q}$ for these two booths. ${ }^{(32)}$ The air changes per min (ACM) were calculated by dividing CFM by the room volume.

\section{RESULTS}

\section{Product Identification}

A total of 29 different isocyanate-containing products from 11 manufacturers were sampled. Clearcoat products were the most frequently sampled, representing $72 \%$ of the collected air samples. Single-stage products, in which a color basecoat is combined with the clearcoat, represented $20 \%$ of the samples. The remaining air samples were collected during the 
application of sealers ( $5 \%$ of samples), pigments $(<1 \%)$, and basecoats $(<1 \%)$. The most frequently used products were manufactured by PPG Industries (29\% of the total) followed by DuPont $(28 \%)$ and BASF $(11 \%)$.

\section{Personal Isocyanate Exposures and Respiratory Protection}

A total of 228 personal breathing zone samples were collected. Quantile-quantile (Q-Q) plots, Shapiro-Wilk tests, and skewness coefficients revealed that the distributions of all analytes were non-normal ( $\mathrm{p} \leq 0.05$ ) and positively skewed. In $26 \%$ of samples, monomeric HDI was below the LOD (Table IV). In $92 \%$ of the samples, monomeric IPDI was below the LOD; consequently, air concentrations of monomeric IPDI were not calculated. HDI isocyanurate and IPDI polyisocyanate were present at the highest concentrations, with only $4 \%$ and $26 \%$ of the samples below their respective LODs. HDI uretdione and HDI biuret concentrations were below their LODs in $55 \%$ and $65 \%$ of the samples, respectively.

Variability Statistics and Probability of Overexposure-Within- and betweenworker variability was calculated for HDI isocyanurate (Table V). The within- and betweenspray painter variance for painting tasks was low at approximately one or less. The probability of overexposure to HDI isocyanurate was tested with the hypothesis: $\mathrm{H}_{\mathrm{O}}: \theta \geq A$, where the probability $(\theta)$ that a randomly chosen worker's mean exposure is greater than the STEL $_{15 \text { min }}$ at a predetermined acceptable probability $(A)$; where $A=0.10$. In this case, the Wald-type test statistic $\hat{W}=9.76$ (Table $\mathrm{V}$ ) exceeds $\mathrm{z}_{0.05}=-1.645$. This suggests that $\theta \geq A$, or that the probability (with $5 \%$ statistical error) that a randomly chosen worker's mean exposure is greater than the OR-OSHA STEL 15 min for HDI polyisocyanates, is larger than the predetermined acceptable probability (i.e., unacceptable exposure). Similarly, when measurements are expressed in units of $\mu \mathrm{g} \mathrm{NCO} / \mathrm{m}^{3}$ and compared with the UK-HSE STEL $_{15 \min }$ for all isocyanates ( $\hat{W}=15.01$; Table V), we conclude that a worker's mean exposure is greater than the UK-HSE STEL $15 \mathrm{~min}$.

Exposures Compared with ACGIH-TLV Excursion Limits-Short-term exposures to substances that have 8-hr TLV-TWAs but no formal STEL established should be controlled. Excursion limits, set at three times the value of the TLV-TWA, apply to monomeric HDI and monomeric IPDI, which have no established STEL. The excursion limits for monomeric HDI and monomeric IPDI are $102 \mu \mathrm{g} / \mathrm{m}^{3}\left(3 \times 34 \mu \mathrm{g} / \mathrm{m}^{3} \mathrm{TLV}\right)$ and 137 $\mu \mathrm{g} / \mathrm{m}^{3}\left(3 \times 34 \mu \mathrm{g} / \mathrm{m}^{3} \mathrm{TLV}\right)$, respectively. No samples exceeded these TLV excursion limits for monomeric HDI or monomeric IPDI.

\section{Exposures Compared with STELs and Evaluation of Respiratory Protection-} Concentrations were compared both with the OR-OSHA STEL $15 \mathrm{~min}$ for HDI polyisocyanates (Table II) and the UK-HSE STEL ${ }_{15 \mathrm{~min}}$ for all isocyanates (Table III). Because it is inappropriate to perform extensive statistical modeling when concentrations are imputed for a large percentage of samples, only simple metrics were calculated, such as the range of concentrations, the number of samples exceeding the $\mathrm{STEL}_{15 \mathrm{~min}}$, or the respirator's MUC (Tables II and III). These data illustrate that (1) spray coatings are composed of multiple polyisocyanate species, (2) for each individual polyisocyanate species at least one sample exceeded the OR-OSHA STEL ${ }_{15 \mathrm{~min}}$, and (3) painters' respiratory protection was not 
always adequate. All painters were observed to wear respiratory protection while spray painting. Half-face air-purifying respirators (APR) were worn by $67 \%$ of painters, whereas $20 \%$ wore supplied-air respirators (SAR, Table VI).

OR-OSHA STEL ${ }_{15 \min }$ for HDI Polyisocyanate Expressed as $1000 \mu \mathrm{g} / \mathrm{m}^{\mathbf{3}}$-Table II shows isocyanate exposures compared with the OROSHA STEL $15 \mathrm{~min}$ listed by individual isocyanate species. The OR-OSHA STEL $15 \mathrm{~min}$ was developed based on the toxicity of HDI isocyanurate and HDI biuret. HDI isocyanurate was the predominant species detected with $153(67 \%)$ samples showing isocyanurate exposure in exceedance of the OROSHA STEL $_{15 \mathrm{~min}}$. When isocyanurate exposure is added to biuret exposure, a total of $160(70 \%)$ samples exceed the OR-OSHA STEL $15 \mathrm{~min}$, while 15 (7\%) samples exceed the respirator's MUC (data not shown). When uretdione is added to the sum of isocyanurate and biuret exposure, the number of samples exceeding the OR-OSHA STEL $15 \mathrm{~min}$ and respirator MUC increases to $182(80 \%)$ and 16 (7\%), respectively (Table II, total HDI polyisocyanates). While IPDI is not covered by the OR-OSHA STEL $15 \mathrm{~min}$, it is noteworthy that $15 \%$ of IPDI measurements exceed this STEL (Table II).

Finally, if IPDI exposure is included in the sum of isocyanurate, biuret, and uretdione exposure, the total number of samples exceeding the OR-OSHA STEL $15 \mathrm{~min}$ and respirator MUC further increases to 198 (87\%) and 27 (12\%) samples, respectively (data not shown). Exposures compared with the OR-OSHA STEL ${ }_{15 \mathrm{~min}}$ can underestimate the burden of exposure when painters use formulations containing IPDI, as it is not included in this STEL. The number of painters for whom at least one sample exceeds the MUC of their respirator increases from 7 (21\%) painters when exposure includes isocyanurate, biuret, and uretdione (total HDI polyisocyanate, Table II) to 11 (33\%) painters when IPDI is included in the total exposure (data not shown).

UK-HSE STEL ${ }_{15 \mathrm{~min}}$ for All Isocyanates Expressed as $70 \mu \mathrm{g} \mathrm{NCO} / \mathrm{m}^{3}$-The comparison of exposures against the UK-HSE STEL ${ }_{15 \mathrm{~min}}$ reveals the same trends as that for the OR-OSHA STEL $15 \mathrm{~min}$. However, because the UK-HSE STEL ${ }_{15 \mathrm{~min}}$ is more conservative, a greater number of measurements exceeded the standard, and a higher fraction of painters were considered inadequately protected (Table III). As seen in the OROSHA STEL $_{15 \text { min }}$ comparison, HDI isocyanurate was the predominant species in the exposure ( $85 \%$ of measurements exceeded the $\mathrm{STEL}_{15 \mathrm{~min}}$ ) followed by IPDI ( $73 \%$ of measurements exceeded the $\mathrm{STEL}_{15 \mathrm{~min}}$ ). When the individual species were summed for direct comparison to the UK-HSE STEL ${ }_{15 \mathrm{~min}}, 98 \%$ of all isocyanate NCO measurements exceeded the UKHSE STEL ${ }_{15 \min }$ of $70 \mu \mathrm{g} \mathrm{NCO} / \mathrm{m}^{3}$. Thirty percent of the measurements exceeded the MUC of the respirator worn at the time of sampling. Based on the total NCO measured and using the UK-HSE STEL $15 \mathrm{~min}, 67 \%$ of the painters were inadequately protected by their respirator during one or more measurements.

Isocyanate Composition of Air Samples-The isocyanate composition of samples was evaluated based on the air concentrations of each analyte within a sample (Table IV). As expected, the percentage of monomers in the samples was low, with a maximum of $0.6 \%$ and $1.5 \%$ for IPDI monomer and HDI monomer, respectively. The percentage of polyisocyanates in the samples was greater and varied considerably between species. The 
maximum percentage of polyisocyanates in the air samples ranged from 58\% HDI biuret to 96\% HDI isocyanurate. Because these percentage composition data did not conform to normal or log-normal distributions (as determined from Q-Q plots), non-parametric statistical procedures were used in the analysis.

For the polyisocyanate species, correlation analysis (Spear-man's rho) revealed a highly significant inverse relationship between the percentage of HDI isocyanurate and the percentage of HDI uretdione in the air samples (correlation coefficient $=-0.763, p \leq 0.001$ ). Similarly, the inverse relationship between the percentage of HDI isocyanurate and the percentage of IPDI polyisocyanate was also highly significant (correlation coefficient = $0.747, \mathrm{p} \leq 0.001)$.

Box plots were prepared to evaluate the percentage composition of the individual analytes in the air samples derived from each of the 29 products. Visual inspection of these plots suggested that several products were possible outliers in terms of their HDI polyisocyanate content. With a confidence level of $95 \%$, the Kruskal-Wallis test revealed significant differences in the percentage composition of the analytes between products. A post-hoc evaluation was then conducted using a Mann-Whitney test of all product pairs, and a Bonferroni adjustment was used to account for multiple comparison error. This analysis revealed that two products had statistically significantly higher percentages of HDI uretdione and lower percentages of HDI isocyanurate than most of the other products ( $\mathrm{p} \leq$ 0.0017). Both products were manufactured by the same company. We contacted the manufacturer to explain the rationale for these differences and were informed that paint formulations are considered proprietary information.

\section{Booth Ventilation}

Ventilation was evaluated in 36 booths in 25 shops (Table VII). The majority of the booths were downdraft, with an average exhaust velocity of approximately 130 LFM (40 LMM) and volumetric airflow of approximately $8800 \mathrm{CFM}\left(250 \mathrm{~m}^{3} / \mathrm{min}\right)$. Air velocity in the painter's breathing zone was not documented in this study. The International Fire Code and the ACGIH require that booths be maintained with an average air velocity over the open face of the booth or booth cross section at or above 100 LFM (40 LMM). ${ }^{(32,33)}$ Although all seven of the semi-downdraft booths met this requirement, $52 \%$ of downdraft booths and none of the cross draft booths met this requirement. Some booth manufactures specify booth performance in terms of air changes per minute, and the average ACM was 3.0 for the downdraft booths. Within-booth performance variability was evaluated over the course of this 7-month study; 22 of the 36 booths were evaluated on three separate visits. Four of these booths exhibited a coefficient of variation (CV) of less than $10 \%$ while the majority $(\mathrm{N}=$ 14) had a CV between 10 and 30\% (Table VIII).

\section{DISCUSSION}

One objective of this study was to evaluate isocyanate exposure in automotive spray painters. We determined that exposures to spray coatings were composed of one monomer (HDI monomer) and a variable mixture of four different polyisocyanates (IPDI polyisocyanate, HDI biuret, HDI uretdione, and HDI isocyanurate). In addition, we 
determined that significant limitations exist in the use of the current STELs for these mixed isocyanate exposures. These findings illustrate the need for OELs that address all polyisocyanate species.

\section{Variability in the Polyisocyanate Species Comprising the Exposure}

Because the analytical method used identified individual isocyanate species, it was possible to calculate the percentage fraction of individual isocyanates within a sample. The minimum percentage of IPDI polyisocyanate, HDI biuret, HDI uretdione, and HDI isocyanurate in a given sample ranged between $\approx 0$ to $4 \%$, while the maximum percent ranged from $58 \%$ to $96 \%$. We conclude, therefore, that there is considerable variability in the possible isocyanate composition among the 29 paint formulations produced by the 11 manufactures investigated here.

While several of these individual isocyanates were predominantly measured at levels below their respective LODs or LOQs, they were measured occasionally at concentrations that exceeded the STELs. Further, as individual species, they were measured at concentrations that may have exceeded the respiratory protection used by the painter. Because HDI isocyanurate was the predominant species, it may be tempting to base exposure assessment on this species alone. However, the paint formulation analysis presented here identified two products very low in HDI isocyanurate but very high in HDI uretdione. Similarly, there was an inverse relationship between the presence of HDI isocyanurate and IPDI polyisocyanate. It is reasonable to expect that paint formulations will change over time and that not all the isocyanate species are recorded on the product's MSDS. Consequently, it is important to quantify all possible isocyanate species in the spray paint when conducting exposure assessment for these mixtures.

Very few samples contained HDI isocyanurate below the LOD. The within- and betweenworker variability was calculated for this species and observed to be low, indicating that the spray painters were exposed to HDI isocyanurate in a homogenous manner. This homogeneity may reflect the fact that the samples were collected only during spray painting operations. The inclusion of other tasks such as paint mixing or vehicle taping would likely introduce more variability in the data.

The Wald-type statistic, based on measurements of HDI isocyanurate, was very high and indicates overexposure (i.e., the probability that a painter's mean exposure is greater than either the OR-OSHA or UK-HSE STELs is larger than the predetermined acceptable probability). Although identifying overexposure to HDI isocyanurate is valuable, all painters wore some type of respiratory protection, which must be incorporated into their personal exposure assessment. Simple counts and percentage calculations of the number of samples exceeding the $\mathrm{STEL}_{15 \mathrm{~min}}$ or the respirator's calculated MUC were therefore described for all of the individual isocyanate species.

\section{Comparison with STELs and Their Limitations}

Exposures were compared with the two most applicable STELs: the OR-OSHA HDI polyisocyanate $\operatorname{STEL}_{15 \min }\left(1000 \mu \mathrm{g} / \mathrm{m}^{3}\right)$ and the UK-HSE all isocyanate $\operatorname{STEL}_{15 \min }(70 \mu \mathrm{g}$ 
$\mathrm{NCO} / \mathrm{m}^{3}$ ). Applying the OR-OSHA STEL 15 min alone to spray painter's exposure may not fully assess exposure risk. Exposure to HDI uretdione should be included in exposure assessment, even though this compound was not part of the toxicity testing that led to the establishment of the OR-OSHA STEL 15 min for HDI polyisocyanates. Exposure to IPDI should be accounted for, as $15 \%$ of samples had concentrations of IPDI that exceeded the OR-OSHA STEL $15 \mathrm{~min}$.

The UK-HSE STEL ${ }_{15 \min }$ standard assumes equivalent toxicity between monomeric and polymeric isocyanates. ${ }^{(34)}$ We noted that $98 \%$ of samples exceeded the UK-HSE STEL $15 \mathrm{~min}$ for all isocyanates. Similarly, Woskie et al. ${ }^{(35)}$ reported that $79 \%$ of HDI polyisocyanate exposures for spray operations in collision repair shops exceeded the UK-HSE STEL ${ }_{15 \mathrm{~min}}$. Applying the UK-HSE STEL $15 \mathrm{~min}$, which is based on the toxicity of the monomer, may not be appropriate for the data presented here because polyisocyanates are the predominant species, rather than monomers. Differences between polyisocyanates and monomeric isocyanates are discussed by Bello et al. ${ }^{(23)}$ Unfortunately, the differences in toxicity are not well understood, in part because polyisocyanates have not been investigated as extensively as the monomers.

High breathing-zone concentrations of several individual polyisocyanates were documented, illustrating the need for polyisocyanates to be sampled comprehensively, analyzed, and compared with comprehensive OELs. A hypothetical comprehensive polyisocyanate OEL, expressed as $\mu$ g total polyisocyanate $\mathrm{NCO} / \mathrm{m}^{3}$, would address all of the possible polyisocyanates found in automotive paint formulations. The advantages of a comprehensive polyisocyanate OEL, expressed as total NCO, are (1) no prior knowledge of the individual isocyanates present in a formulation would be required (the isocyanate composition of a product may be proprietary and not available from MSDSs); (2) changes in paint formulations would be accommodated, and (3) investigators could still compare monomer concentrations with their respective monomer OELs. The greatest limitation to a comprehensive polyisocyanate $\mathrm{NCO} / \mathrm{m}^{3}$ OEL is that the differences in toxicity between the individual polyisocyanates are not accounted for. ${ }^{(24)}$ While this is a significant limitation, the alternative of developing OELs for all possible individual polyisocyanates would be challenging. The need for a comprehensive polyisocyanate OEL has been suggested previously by Bello et al. ${ }^{(23)}$ and the results reported here demonstrate this necessity.

The majority of painters enrolled in this study $(67 \%)$ wore either negative pressure half-face respirator (APRs) with replaceable filters or disposable half-face APRs. This observation is consistent with a Washington State industry-wide collision repair survey, in which 69\% of shops reported that painters wore half-face APRs when spraying two-part clearcoats. ${ }^{(10,11)}$ Sparer et al. ${ }^{(2)}$ reported that $86 \%$ of Connecticut shops provided half-face APRs to spray painters. Using the OR-OSHA and UK-HSE STEL metrics, we conclude that $21 \%$ and $63 \%$ (respectively) of the painters in this study were not adequately protected against polyisocyanates when wearing half-face APRs having an OSHA APF of 10. The concentrations documented in this study compared with the OR-OSHA STEL $15 \mathrm{~min}$ suggest that painters should use respirators with an APF of 25 or greater, such as full-face APRs, powered air-purifying respirators (PAPRs), or any supplied air respirators operated in 
continuous flow mode. Full-face respirators have the additional advantage of providing eye protection.

Liu et al. ${ }^{(36)}$ used a PortaCount Plus respirator fit tester to calculate workplace protection factors and concluded that half-face APRs provided effective protection against isocyanates in properly trained and fitted workers. However, respirator training and fit testing is a challenge for an industry where only $25 \%$ report having contracted with a safety and health consultant. ${ }^{(10,11)}$ The use of SARs when applying auto paints has been recommended by NIOSH, the U. S. Environmental Protection Agency, and some paint manufacturers. Airpurifying half-face respirators are unlikely to protect against intense overspray conditions (we observed a two-person spraying operation in which painters were inadvertently spraying each other) or when painting large objects such as buses, boats, or trailers. ${ }^{(21)}$

Because respirators are not always worn and maintained properly, adequate exhaust ventilation is equally important as it can reduce the burden of exposure. While all seven of the semi-downdraft booths in this study met an average of 100 LFM exhaust velocity, only $52 \%$ of the downdraft booths and none of the four cross-draft booths met this target. The ventilation measurements presented here are discussed by co-authors in Fent et al. ${ }^{(24)}$ and shown to be correlated with polyisocyanate breathing zone concentrations. Increased airflow and downdraft booths (vs. cross- or semi-draft booths) were associated with reduced polyisocyanate breathing zone concentrations. Gaines et al. ${ }^{(37)}$ and Flack et al. ${ }^{(38)}$ documented that the type of spray booth was a significant predictor of HDA level $(1,6-$ hexamethylene diamine, an HDI biomarker) in the urine and blood plasma of autobody spray painters, regardless of the painters' choice of respiratory protection. Education and outreach are needed to improve respiratory protection and spray booth ventilation in the collision repair industry.

The strengths of this study include comprehensive sampling and analysis of all isocyanatecontaining coatings used throughout a spray painter's workday, sampled up to three times over several months. While not all analytical methods identify specific isocyanate species, the analytical method described here identified all species currently known to be present in automotive paints, including IPDI. This allowed us to describe the percent fraction of individual species within the air samples.

Limitations of this study include the loss of subject follow-up due to the length of the study. In addition, because shops participated in this study on a voluntary basis, they may not have been representative of the industry as a whole. Participating shops may have used different paint lines or had different safety and health programs compared with non-participating shops. As mentioned previously, the prevalence of half-face respirators in this study population was nearly identical to that reported by Washington shops in a 2006 industrywide survey. ${ }^{(10,11)}$

In addition, the median (1) and average (1.4) number of painters per shop was very similar to that reported in the 2006 survey (median of 1 and an average of 1.8 painters). ${ }^{(10,11)}$ The paint products documented in this study were also similar to those reported in the 2006 industry survey. In these respects, we conclude that the study population was representative 
of all collision repair shops in Washington. The air sampling conducted here was specific to the task of spray painting and based on the spray time, which was $40 \%$ of the total sampling time. Concentrations based on total sampling time would be lower than those presented here. Exposures to isocyanates during other tasks, such as paint mixing, were not evaluated.

\section{CONCLUSION}

The isocyanate exposures documented in this collision repair study are a mixture of HDI monomer and four additional polyisocyanates. Comparison of these mixed exposures with existing STELs is challenging. An OEL should be established that is comprehensive for polyisocyanates, perhaps similar to the OR-OSHA STEL 15 min for HDI polyisocyanates. Alternatively, consensus or guidance regarding the applicability of the UK-HSE STEL $15 \mathrm{~min}$ (based on monomeric toxicity) to mixed polyisocyanate exposures is needed.

While several painters used supplied-air respirators in this study, the predominant choice of protection was a half-face air-purifying respirator. Despite the limitations of the OR-OSHA STEL $_{15 \mathrm{~min}}$, we estimated that isocyanate exposures in the collision repair industry require respirators having an APF of 25 or greater. In addition to respiratory protection, effective exhaust ventilation and dermal protection are required to reduce the risk of exposure to isocyanates.

\section{Acknowledgments}

We gratefully acknowledge the spray painters and auto-body shop owners who participated in this study. Guidance and support from automotive industry associations as well as local paint training facilities were gratefully appreciated. Thanks to chemist Wally Suydam of the Washington State DOSH analytical laboratory. We acknowledge the statistical expertise provided by Tom Georgianna (King County Department of Natural Resources and Parks, Office of Water and Land Resources). Thanks to Dr. Mike Yost of the University of Washington's Exposure Sciences Program for his support. Funding was provided by the SHARP Program at the Washington State Department of Labor and Industries, National Institute for Occupational Safety and Health (R01-OH007598 and T42OH008673), and National Institute of Environmental Health Sciences (T32 ES007018).

\section{REFERENCES}

1. National Institute for Occupational Safety and Health (NIOSH). Preventing Asthma and Death from Diisocyanate Exposure (DHHS/NIOSH Pub. No. 96-111). NIOSH; Cincinnati, Ohio: 1996.

2. Sparer J, Stowe MH, Bello D, et al. Isocyanate exposures in autobody shop work: The SPRAY study. J. Occup. Environ. Hyg. 2004; 1:570-581. [PubMed: 15559329]

3. Bello D, Sparer J, Redlich CA, Ibrahim K, Stowe MH, Liu Y. Slow curing of aliphatic polyisocyanate paints in automotive refinishing: A potential source for skin exposure. J. Occup. Environ. Hyg. 2007; 4:406-411. [PubMed: 17474030]

4. Liu Q, Wisnewski AV. Recent developments in diisocyanate asthma. Ann. Allergy Asthma Immunol. 2003; 90(5 Suppl 2):35-41. [PubMed: 12772950]

5. Petsonk EL, Wang ML, Lewis DM, Siegel PD, Husberg BJ. Asthma-like symptoms in wood product plant workers exposed to methylene diphenyl diisocyanate. Chest. 2000; 118(4):11831193. [PubMed: 11035694]

6. Redlich CA, Herrick HA. Lung/skin connections in occupational lung disease. Curr. Opin. Allergy Clin. Immunol. 2008; 8:115-119. [PubMed: 18317018]

7. Donnelly R, Buick JB, Macmahon J. Occupational asthma after exposure to plaster casts containing methylene diphenyl diisocyanate. Occup. Med. (Lond). 2004; 54(6):432-434. [PubMed: 15358843]

8. Bernstein JA. Overview of diisocyanate occupational asthma. Toxicology. 1996; 111:181-189. [PubMed: 8711734] 
9. Pronk A, Preller L, Raulf-Heimsoth M, et al. Respiratory symptoms, sensitization, and exposure response relationships in spray painters exposed to isocyanates. Am. J. Respir. Crit. Care Med. 2007; 176(11):1090-1097. [PubMed: 17656675]

10. Whittaker SG, Reeb-Whitaker C. Characterizing the health and safety needs of the collision repair industry. J. Occup. Environ. Hyg. 2009; 6:273-282. [PubMed: 19235620]

11. Whittaker, SG.; Anderson, R.; Whitaker, C. Health and Safety in Washington State's Collision Repair Industry: A Needs Assessment (Technical Report No. 69-4-2005). Washington State Department of Labor \& Industries; Olympia, Wash: 2005.

12. Balmes J, Becklake M, Blanc P, et al. American Thoracic Society Statement: Occupational contribution to the burden of airway disease. Am. J. Respir. Crit. Care Med. 2003; 167(5):787797. [PubMed: 12598220]

13. Bakerly ND, Moore VC, Vellore AD, Jaakkola MS, Robertson AS, Burge PS. Fifteen-year trends in occupational asthma: data from the Shield surveillance scheme. Occup. Med. (Lond). 2008; 58(3):169-174. [PubMed: 18308695]

14. Curwick, C.; Bonauto, D. Work-Related Asthma in Washington State: A Review of Worker's Compensation Claims from 1995-2002 (Technical Report No. 64-6-2003). Washington State Department of Labor and Industries; Olympia, Wash: 2003.

15. "Occupational asthma. Causal agents for occupational asthma." [Online]. Available at www.hse.gov.uk/statistics/causdis/asthma (accessed March 23, 2012)

16. Erjefalt I, Persson CG. Increased sensitivity to toluene diisocyanate (TDI) in airways previously exposed to low doses of TDI. Clin. Exp. Allergy. 1992; 22(9):854-862. [PubMed: 1330235]

17. Herrick CA, Xu LX, Wisnewski AV, Das J, Redlich CA, Bottomly K. A novel mouse model of diisocyanate-induced asthma showing allergic-type inflammation in the lung after inhaled antigen challenge. J. Allergy Clin. Immunol. 2002; 109(5):873-878. [PubMed: 11994714]

18. Pauluhn J. Brown Norway rat asthma model of diphenylmethane 4,4'-diisocyanate. Inhal. Toxicol. 2005; 17(13):729-739. [PubMed: 16195208]

19. Rattray NJ, Botham PA, Hext PM, et al. Induction of respiratory hypersensitivity to diphenylmethane-4,4'-diisocyanate (MDI) in guinea pigs. Influence of route of exposure. Toxicology. 1994; 88(1-3):15-30. [PubMed: 8160196]

20. Ferguson JS, Schaper M, Alarie Y. Pulmonary effects of a polyisocyanate aerosol: Hexamethylene diisocyanate trimer (HDIt) or Desmodur-N (DES-N). Toxicol. Appl. Pharmacol. 1987; 89(3):332346. [PubMed: 3603565]

21. Janko M, McCarthy K, Fajer M, van Raalte J. Occupational exposure to 1,6-hexamethylene diisocyanate-based polyisocyanates in the state of Oregon, 1980-1990. Am. Ind. Hyg. Assoc. J. 1992; 53:331-338. [PubMed: 1609744]

22. Health and Safety Executive (HSE). HSE Review 1981. HSE; Bootle, U.K.: 1993. p. C18

23. Bello D, Woskie SR, Streicher RP, et al. Polyisocyanates in occupational environments: A critical review of exposure limits and metrics. Am. J. Ind. Med. 2004; 46(5):480-491. [PubMed: 15490474]

24. Fent KW, Gaines LG, Thomasen JM, et al. Quantification and statistical modeling-Part I: Breathing-zone concentrations of monomeric and polymeric 1,6-hexamethylene diisocyanate. Ann. Occup. Hyg. 2009; 53(7):677-689. [PubMed: 19622637]

25. Ceballos DM, Fent KW, Whittaker SG, et al. Survey of dermal protection in Washington State collision repair industry. J. Occup. Environ. Hyg. 2010; 8:551-560. [PubMed: 21830873]

26. Division of Occupational Safety and Health (DOSH) Lab, WA State Department of Labor \& Industries. Method L\&I 0067, "HDI and IPDI Polyisocyanate Aerosol.". Washington State Department of Labor \& Industries, rev.; Olympia, Wash: 2008.

27. Division of Occupational Safety and Health (DOSH) Lab, WA State Department of Labor \& Industries. Method L\&I 0050 "HDI, IPDI and MDI (Vapor) MAMA Derivatives,". Washington State Department of Labor \& Industries, rev.; Olympia, Wash: 2008.

28. Ceballos DM, Whittaker SG, Yost MG, et al. A laboratory comparison of analytical methods used for isocyanates. Anal. Methods. 2011; 3(11):2478-2487.

29. Hornung RW, Reed LD. Estimation of average concentration in the presence of nondetectable values. Appl. Occup. Environ. Hyg. 1990; 5:46-51. 
30. "Respiratory Protection, Personal Protective Equipment,". Code of Federal Regulations Title 29. 1910:134. Part.

31. Rappaport SM, Lyles RH, Kupper LL. An exposure-assessment strategy accounting for withinand between-worker sources of variability. Ann. Occ. Hyg. 1995; 39(4):469-495.

32. ACGIH: Ventilation: A Manual of Recommended Practice. 25th Edition. ACGIH; Cincinnati, Ohio: 2004.

33. International Code Council (ICC). International Fire Code. ICC; Washington, D.C.: 2006. Air velocity.. Chapter 15, Section 1504.7.3.

34. Silk SJ, Hardy HL. Control limits for isocyanates. Ann. Occup. Hyg. 1983; 27(4):333-339.

35. Woskie SR, Sparer J, Gore RJ, et al. Determinants of isocyanate exposures in auto body repair and refinishing shops. Ann. Occup. Hyg. 2004; 48(5):393-403. [PubMed: 15148052]

36. Liu Y, Stowe MH, Bello D, et al. Respiratory protection from isocyanate exposure in the autobody repair and refinishing industry. J. Occup. Environ. Hyg. 2006; 3:234-249. [PubMed: 16574607]

37. Gaines LGT, Fent KW, Flack SL, Thomasen JM, Whittaker SG, Nylander-French LA. Factors affecting variability in the urinary biomarker 1,6-hexamethylene diamine in workers exposed to 1,6-hexamethylene diisocyanate. J. Environ. Monit. 2011; 13(1):119-127. [PubMed: 20978689]

38. Flack SL, Fent KW, Gaines LGT, et al. Quantitative plasma biomarker analysis in HDI exposure assessment. Ann. Occup. Hyg. 2010; 54(1):41-54. [PubMed: 19805392] 


\section{TABLE 1}

Calibration Standards and Analysis Parameters for the Isocyanate Analytical Method

\begin{tabular}{|c|c|c|c|c|c|}
\hline Analyte & Standard & $\operatorname{LOD}(\mu$ g/filter $)$ & LOQ ( $\mu$ g/filter) & Recovery (\%) & Precision $(\% \mathrm{CV})$ \\
\hline HDI monomer & Omega calibration standard ${ }^{A}$ & 0.005 & 0.11 & 93.8 & 0.13 \\
\hline IPDI monomer & Omega calibration standard ${ }^{A}$ & 0.004 & 0.13 & 91.7 & 0.11 \\
\hline HDI uretdione & Bayer Desmodur 3400-N & 2.8 & 4.8 & 158.6 & 0.22 \\
\hline HDI biuret & Bayer Desmodur N100 & 1.7 & 1.9 & 87.8 & 0.13 \\
\hline HDI isocyanurate & Bayer Desmodur N3300A & 1.2 & 5 & 107.6 & 0.15 \\
\hline IPDI polyisocyanate & Bayer Desmodur Z4470-BA & 1.7 & 15.7 & 98.4 & 0.19 \\
\hline
\end{tabular}

${ }^{A}$ Calibration standard set-Cat\# 225-9055, Omega Specialty Instrument Co, Houston, Texas. 


\section{TABLE II}

Isocyanate Air Concentrations Expressed as $\mu \mathrm{g} / \mathrm{m}^{3} \mathrm{STEL}_{15 \mathrm{~min}}$ and Compared with the OR-OSHA HDI Polyisocyanate $\operatorname{STEL}_{15 \mathrm{~min}}\left(1000 \mu \mathrm{g} / \mathrm{m}^{3}\right)$ and the Calculated Respirator Maximum Use Concentration (MUC)

\begin{tabular}{|c|c|c|c|c|c|}
\hline Analyte & $\mathbf{N}$ & Range $\mu \mathrm{g} / \mathrm{m}^{3}$ & Samples $(\%)>1000 \mu \mathrm{g} / \mathrm{m}^{3}$ & Samples $(\%)>M_{U} \mathbf{C}^{A}$ & Painters $(\%)>M_{U C} B$ \\
\hline HDI monomer $C$ & 224 & $0-30$ & na & na & na \\
\hline IPDI polyisocyanate ${ }^{C}$ & 228 & $70-11,212$ & 15 & 1 & 6 \\
\hline HDI uretdione & 227 & $68-24,986$ & 14 & 2 & 9 \\
\hline HDI biuret & 228 & $37-1945$ & 3 & 0 & 0 \\
\hline HDI isocyanurate & 228 & $51-20,143$ & 67 & 5 & 12 \\
\hline Total HDI polyisocyanate ${ }^{D}$ & 228 & $240-29,789$ & 80 & 7 & 21 \\
\hline \multicolumn{6}{|l|}{ Note: $\mathrm{N}=$ number of samples. } \\
\hline \multicolumn{6}{|c|}{${ }^{A}$ OSHA-assigned APF x $1000 \mu \mathrm{g} / \mathrm{m}^{3}$. } \\
\hline \multicolumn{6}{|c|}{${ }^{B}$ Percentage of painters for which one or more samples exceeded the respirator MUC ( $\mathrm{n}=33$ painters). } \\
\hline
\end{tabular}




\section{TABLE III}

Isocyanate Air Concentrations Expressed as $\mu \mathrm{g} \mathrm{NCO} / \mathrm{m}^{3} \mathrm{STEL}_{15 \mathrm{~min}}$ Compared with the UK-HSE All isocyanate $\mathrm{STEL}_{15 \mathrm{~min}}$ of $70 \mu \mathrm{g} \mathrm{NCO} / \mathrm{m}^{3}$ and the Calculated Respirator MUC

\begin{tabular}{|c|c|c|c|c|c|}
\hline Analyte & $\mathbf{N}$ & Range NCO $\mu \mathrm{g} / \mathrm{m}^{3}$ & $\begin{array}{c}\text { Samples }(\%)>70 \mu g \\
\mathrm{NCO} / \mathrm{m}^{3}\end{array}$ & Samples (\%) >MUC ${ }^{A}$ & Painters $(\%)>$ MUC $^{B}$ \\
\hline HDI monomer & 224 & $0-15$ & 0 & 0 & 0 \\
\hline IPDI polyisocyanate & 228 & $12-1936$ & 73 & 3 & 9 \\
\hline HDI uretdione & 227 & $15-5408$ & 30 & 5 & 15 \\
\hline HDI biuret & 228 & $8-427$ & 14 & 0 & 0 \\
\hline HDI isocyanurate & 228 & $11-4341$ & 85 & 20 & 52 \\
\hline Total HDI polyisocyanate ${ }^{C}$ & 228 & $52-6444$ & 97 & 27 & 55 \\
\hline Total all isocyanate $\mathrm{NCO}^{D}$ & 228 & $65-5174$ & 98 & 30 & 67 \\
\hline
\end{tabular}

Note: UK-HSE STEL15min applies to any isocyanate.

${ }^{A}$ OSHA-assigned APF x $70 \mu \mathrm{g} / \mathrm{m}^{3} \mathrm{NCO}$.

${ }^{B}$ Percentage of painters for which one or more samples exceeded the respirator MUC $(\mathrm{n}=33)$.

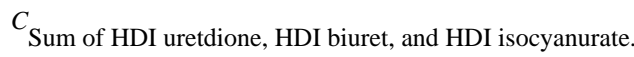

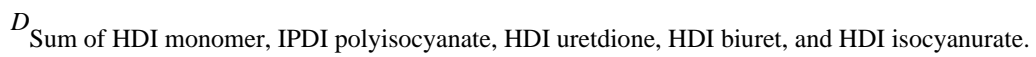




\section{TABLE IV}

Air Sample Data

\begin{tabular}{lcccccc}
\hline & & & & \multicolumn{2}{c}{$\begin{array}{c}\text { Range of Isocyanate Composition (\%) Within } \\
\text { Air Samples }\end{array}$} \\
\cline { 5 - 7 } Analyte & $\mathbf{N}$ & $\mathbf{N}(\%)<\mathbf{L O D}$ & $\mathbf{L O D}<\mathbf{N}(\%)<\mathbf{L O Q}$ & $\mathbf{N}(\%)>\mathbf{L O Q}$ & $\mathbf{M i n}$ & Max \\
\hline HDI monomer & 224 & $58(26)$ & $106(47)$ & $60(27)$ & 0.0 & 1.5 \\
IPDI monomer & 223 & $206(92)$ & $17(8)$ & $0(0)$ & 0.0 & 0.6 \\
IPDI polyisocyanate & 228 & $59(26)$ & $134(59)$ & $35(15)$ & 1.2 & 78 \\
HDI uretdione & 227 & $125(55)$ & $25(11)$ & $77(34)$ & 0.3 & 66 \\
HDI biuret & 228 & $149(65)$ & $3(1)$ & $76(33)$ & 0.2 & 58 \\
HDI isocyanurate & 228 & $10(4)$ & $22(10)$ & $196(86)$ & 3.8 & 96 \\
\hline
\end{tabular}

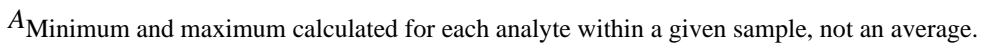


TABLE V

Variability Statistics for HDI Isocyanurate and Comparison with the STEL

\begin{tabular}{llccccccc}
\hline Analyte & Units & $\mathbf{N}$ & $\boldsymbol{\mu}_{\boldsymbol{y}} \boldsymbol{A}$ & $\sigma^{\mathbf{2}}{ }_{\mathrm{w}} \boldsymbol{A}$ & $\boldsymbol{\sigma}^{\mathbf{2}}{ }_{\mathbf{b}} \boldsymbol{A}$ & $\boldsymbol{\mu}_{\mathbf{x}} \boldsymbol{B}$ & $\mathbf{S T E L}$ & $\hat{W}^{\boldsymbol{C}}$ \\
\hline HDI Isocyanurate & $\mu \mathrm{gg} / \mathrm{m}^{3}$ & 228 & 7.332 & 1.062 & 0.787 & 3851.61 & $1000^{D}$ & 9.76 \\
HDI Isocyanurate & $\mu \mathrm{gNCO} / \mathrm{m}^{3}$ & 228 & 5.797 & 1.062 & 0.787 & 830.09 & $70^{E}$ & 15.01 \\
\hline
\end{tabular}

${ }_{\mu y,}^{A} \sigma_{\mathrm{W}}$ and $\sigma^{2} \mathrm{~b}$ are the estimates of the mean, variance within-workers, and variance between-workers, respectively. Exposure concentrations were natural log-transformed.

${ }^{B}$ Estimate of the mean exposure; $\left.\mu_{\boldsymbol{x}}=\exp \left(\mu_{\mathrm{y}}+\left[\sigma^{2} \mathrm{w}+\sigma^{2} \mathrm{~b}\right) / 2\right]\right)$.

$C_{\text {Estimate of the Wald-type statistic. }}$

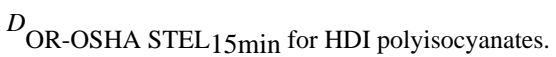

$E_{\text {UK-HSE STEL } 15 \mathrm{~min} \text { for all isocyanates. }}$ 


\section{TABLE VI}

Respirators Used by the Painters

\begin{tabular}{lc}
\hline Respirator Type & Painters (\%) \\
\hline Half-face air-purifying respirator with replaceable filters & 44 \\
Disposable half-face air-purifying respirator & 23 \\
Supplied-air respirator with a helmet or hood & 15 \\
Powered air-purifying respirator (PAPR) & 10 \\
Supplied-air half-face respirator & 5 \\
Full-face air-purifying respirator with replaceable filters & 4 \\
\hline
\end{tabular}

J Occup Environ Hyg. Author manuscript; available in PMC 2014 June 30. 


\section{TABLE VII}

Ventilation by Booth Type

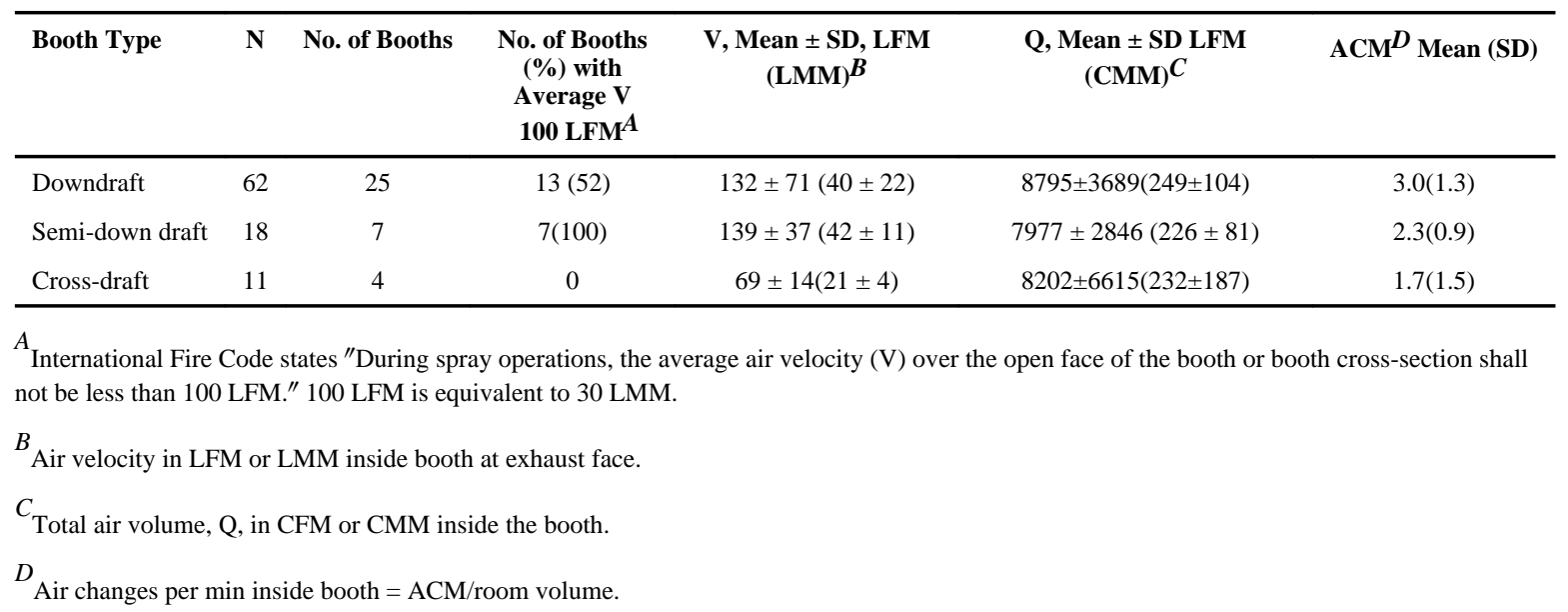




\section{TABLE VIII}

CV in Booth Velocity for Booths Evaluated on Three Separate Occasions During the 7-Month Study Period

\begin{tabular}{lcccc}
\hline Booth Type & No. of Booths & Booths with CV $<\mathbf{1 0 \%}$ & Booths with CV 10-30\% & Booths with CV $>\mathbf{3 0 \%}$ \\
\hline Downdraft & 15 & 3 & 9 & 3 \\
Semi-downdraft & 4 & 0 & 3 & 1 \\
Cross-draft & 3 & 1 & 2 & 0 \\
\hline
\end{tabular}

